PROCEEDINGS OF THE

AMERICAN MATHEMATICAL SOCIETY

Volume 125, Number 1, January 1997, Pages 1-8

S 0002-9939(97)03841-0

\title{
A FORMULA WITH NONNEGATIVE TERMS FOR THE DEGREE OF THE DUAL VARIETY OF A HOMOGENEOUS SPACE
}

\author{
CARRADO DE CONCINI AND JERZY WEYMAN
}

(Communicated by Eric M. Friedlander)

\begin{abstract}
Let $G$ be a reductive group and $P$ a parabolic subgroup. For every $P$-regular dominant weight $\lambda$ let $X(\lambda)$ denote the variety $G / P$ embedded in the projective space by the embedding corresponding to the ample line bundle $\mathcal{L}(\lambda)$. Writing $\lambda=\rho_{P}+\sum_{i=1}^{n} m_{i}^{\prime} \omega_{i}$, we prove that the degree $d(\lambda)^{\vee}$ of the dual variety to $X(\lambda)$ is a polynomial with nonnegative coefficients in $m_{1}^{\prime}, \ldots, m_{n}^{\prime}$. In the case of homogeneous spaces $G / B$ we find an expression for the constant term of this polynomial.
\end{abstract}

\section{INTRODUCTION}

Let $X$ be a nonsingular projective variety of dimension $l$ embedded in the projective space $P^{n}$. Let $H$ be the hyperplane section divisor on $X$. We are interested in the degree of the dual variety $X^{\vee}$ contained in the dual projective space $\left(P^{N}\right)^{\vee}$. There are various formulas for this degree, notably the formula in terms of $H$ and the Chern classes of a sheaf of differentials of $X$ (due to Kleiman, cf. [Kl]), and in terms of Cayley-Koszul type complexes (cf. [G-K-Z], [W]). The drawback of these formulas is that they give a degree as the alternating sum of positive terms, and therefore it is difficult to decide when the degree is equal to 0 (which means that $X^{\vee}$ has codimension $\geq 2$ ).

Therefore it is desirable to give a formula for the degree which expresses it as a sum of nonnegative terms. The goal of this note is to reformulate Kleiman's formula in such a way.

The contents of the paper are as follows. In section 2 we prove the general result on nonnegativity. The proof is a simple extension of Katz's proof of Kleiman's formula (cf. $[\mathrm{K}])$. In section 3 we apply our result to the duals of homogeneous spaces $G / P$. We show that in this case it gives the degree as a sum of nonnegative terms. More precisely, if $G / P$ is embedded by a very ample line bundle $\mathcal{L}(\lambda)$ where $\lambda$ is a weight such that $\lambda-\rho_{P}=\sum_{i=1}^{s} m_{i}^{\prime} \omega_{i}$ where $\omega_{i}$ are the fundamental weights corresponding to the generators of Pic $G / P$ (see section 3 for the notation), then the degree $d(\lambda)^{\vee}$ of the dual variety equals $Q\left(m_{1}^{\prime}, \ldots, m_{s}^{\prime}\right)$ where $Q$ is a polynomial with nonnegative coefficients. We apply the formula to give a short proof of one

Received by the editors January 27, 1995.

1991 Mathematics Subject Classification. Primary 13D25, 14N05; Secondary 13D02, 14M15, $15 \mathrm{~A} 72$.

The second author was partially supported by NSF grant \#DMS-9104867.

(C)1997 American Mathematical Society 
of the results of Knop and Mentzel from [K-M]. In section 4 we investigate the constant term of the polynomial $Q$ when $P=B$ is a Borel subgroup in $G$. We find the expression for this constant term in terms of permanents of certain matrices associated to a root system of $G$.

\section{The CONORMAL CONSTRUCtion, Kleiman's FORMula AND POSITIVITy}

We start with some notation. For any smooth projective variety $T$ we will denote by $A(T)$ the Chow ring of $T$. Given $z \in A(T)$ we will denote by

$$
\int_{T} z
$$

the degree of the graded component of $z$ of dimension 0 . We will call this number the degree of $z$. The class in $A(T)$ of an invertible sheaf $\mathcal{L}$ on $T$ will be also denoted by $\mathcal{L}$.

Let $X$ be a nonsingular variety of dimension 1 . Let us consider an independent variable $t$ and the polynomial

$$
P(t)=\sum_{i=0}^{l} c_{l-i}\left(\Omega_{X}^{1}\right) t^{i+1}
$$

We will denote by $P^{(j)}(t)$ the $j$ th derivative of $P(t)$.

Let $\mathcal{L}$ be a very ample line bundle over $X$. Throughout this section we assume that $\mathcal{L}$ can be written

$$
\mathcal{L}=\mathcal{L}_{1}+\cdots+\mathcal{L}_{r}
$$

where $\mathcal{L}_{i}$ are the bundles such that the corresponding linear systems have no base points and such that for each $i$ there is a representative of the linear system corresponding to $\mathcal{L}_{i}$ which is a smooth subvariety of $X$. The bundle $\mathcal{L}$ defines the embedding of $X$ into the projective space $P^{n}$. We denote by $X^{\vee}$ the projectively dual variety to $X$.

Proposition 1. let $X$ and $\mathcal{L}$ be as above. Then for each $j=1, \ldots, l$ and for each monomial $\mathcal{L}_{1}^{u_{1}}, \ldots, \mathcal{L}_{r}^{u_{r}}$ of degree $j$,

$$
\int_{X} P^{(j)}(\mathcal{L}) \cdot \mathcal{L}_{1}^{u_{1}} \cdots \mathcal{L}_{r}^{u_{r}} \geq 0
$$

Proof. The main tool in the proof is the conormal construction. Let $X, \mathcal{L}$ and $X^{\vee}$ be as above and let $I$ be the sheaf of ideals on $P^{n}$ defining $X$. We denote by $N$ the conormal sheaf $I / I^{2}$. This is a locally free sheaf on $X$ of dimension $n-l$. Let $P(N)$ be the projective bundle on $X$ of lines in $N^{\vee}$. The bundle $P(N)$ can be identified with a subvariety of $X \times\left(P^{n}\right)^{\vee}$ consisting of pairs $(x, H)$ such that $H$ is tangent to $X$ at $x$. Let us denote by

$$
\nu: P(N) \rightarrow X \times\left(P^{n}\right)^{\vee}
$$

the embedding of $P(N)$ into $X \times\left(P^{n}\right)^{\vee}$, and by

$$
\phi: P(N) \rightarrow\left(P^{n}\right)^{\vee}
$$

the composition of $\nu$ with the second projection. By definition $X^{\vee}$ is the image of $\phi$. Let us notice that $\nu$ can be given locally in the following form. If $F$ is a local 
section of $I / I^{2}$, then

$$
P\left(I / I^{2}\right)_{x} \ni F \mapsto\left(x, \text { hyperplane } \sum \frac{\partial F}{\partial X_{i}}(x) T_{i}\right) .
$$

Let $Y$ be a smooth subvariety of codimension $y$ in $X$. Let us denote by $P_{Y}$ the total space of $P(N) \mid Y$ and by $Y^{\vee}$ the image $\phi_{*}\left(P_{Y}\right)$.

If $H^{\vee}$ denotes the class of hyperplane section in $A\left(\left(P^{n}\right)^{\vee}\right)$, then by definition

$$
\operatorname{deg}\left(Y^{\vee}\right)=\int_{\left(P^{n}\right)^{\vee}}\left(H^{\vee}\right)^{n-y-1} \cdot\left(Y^{\vee}\right) .
$$

We can rewrite this as

$$
\operatorname{deg}\left(Y^{\vee}\right)=\frac{1}{\operatorname{deg}(\phi)} \int_{\left(P^{n}\right)^{\vee}}\left(H^{\vee}\right)^{n-y-1} \cdot \phi_{*}\left(P_{Y}\right)
$$

where $\operatorname{deg}(\phi)$ denotes the degree of the morphism from $P_{Y}$ to $Y^{\vee}$ induced by $\phi$. Using the projection formula we get

$$
\operatorname{deg}\left(Y^{\vee}\right)=\frac{1}{\operatorname{deg}(\phi)} \int_{P_{Y}}\left(\phi^{*}\left(H^{\vee}\right)\right)^{n-y-1} .
$$

It remains to calculate the class $\phi^{*}\left(H^{\vee}\right)$. Let us denote by $\psi$ the invertible sheaf $O_{P(N)}(1)$ on $P(N)$, and by $\pi$ the structural morphism $\pi: P(N) \rightarrow X$. Then one can see easily that in $A(P(N))$ we have

$$
\phi^{*}\left(H^{\vee}\right)=\psi-\pi^{*}(H) .
$$

Restricting to $P_{Y}$ we can finally write

$$
\operatorname{deg}\left(Y^{\vee}\right)=\frac{1}{\operatorname{deg}(\phi)} \int_{P_{Y}}\left(\phi^{*}\left(H^{\vee}\right)\right)^{n-y-1} .
$$

This means that we can rewrite formula (5) as

$$
\operatorname{deg}\left(Y^{\vee}\right)=\frac{1}{\operatorname{deg}(\phi)} \int_{P_{Y}}\left(\psi-\pi^{*}(H)\right)^{n-y-1} .
$$

Let us recall that the total Chern class of the vector bundle $\mathcal{V}$ of dimension $v$ over a scheme $X$ is $C(\mathcal{V}):=\sum_{i=0}^{v} c_{i}(\mathcal{V})$.

Now we can apply Lemmas 5.3 and 5.4 from $[\mathrm{K}]$ to the variety $Y$ and the bundle $\left.N\right|_{Y}$.

We get the formula

$$
\operatorname{deg}\left(Y^{\vee}\right)=\frac{(-1)^{l+y}}{\operatorname{deg}(\phi)} \int_{Y} \frac{(1+\mathcal{L})^{n-y-1}}{C\left(\left.N\right|_{Y}\right)} .
$$

Let us denote by $C(X)$ the total Chern class of the tangent bundle $T_{X}$. Then we have

$$
C(N) C(X)=\left.C\left(P^{n}\right)\right|_{X}=(1+\mathcal{L})^{n+1} .
$$

Putting these two formulas together gives

$$
\operatorname{deg}\left(Y^{\vee}\right)=\frac{(-1)^{l+y}}{\operatorname{deg}(\phi)} \int_{Y} \frac{\left.C(X)\right|_{Y}}{(1+\mathcal{L})^{y+1}} .
$$


Using the projection formula for the embedding of $Y$ into $X$ we get

$$
\operatorname{deg}\left(Y^{\vee}\right)=\frac{(-1)^{l+y}}{\operatorname{deg}(\phi)} \int_{X} \frac{[Y] C(X)}{(1+\mathcal{L})^{y+1}}
$$

where $[Y]$ denotes the fundamental class of $Y$.

Let us recall that we assumed that $\mathcal{L}=\mathcal{L}_{1}+\cdots+\mathcal{L}_{r}$ where each $\mathcal{L}_{i}$ is base point free, and such that each class $\mathcal{L}_{i}$ in $A(X)$ has a smooth representative. Let us consider the morphism of $X$ to the product $\mathcal{P}$ of projective spaces defined by the bundles $\mathcal{L}_{1}, \ldots, \mathcal{L}_{r}$. By repeatedly applying to $\mathcal{P}$ the Bertini type theorem ([Ha, Ch. III, Theorem 10.8]) we see that for each exponent $u_{1}, \ldots, u_{r}$ there exists a nonsingular subvariety $Y$ in $X$ such that $[Y]=\mathcal{L}_{1}^{u_{1}} \cdots \mathcal{L}_{r}^{u_{r}}$. Applying (11) to such $Y$, we get the statement of the theorem.

The proof of the proposition also has the following consequence.

Corollary 2. Let $X, \mathcal{L}$ and $\mathcal{L}_{1}, \ldots, \mathcal{L}_{r}$ be as in Proposition 1. Let us consider the smooth subvariety $Y$ such that $[Y]=\mathcal{L}_{1}^{u_{1}} \cdots \mathcal{L}_{r}^{u_{r}}$. Let us assume that $\operatorname{dim} \phi_{*}\left(P_{Y}\right)=$ $\operatorname{dim} P_{Y}$. Then the number

$$
\int_{X} P^{(j)}(\mathcal{L}) \cdot \mathcal{L}_{1}^{u_{1}} \cdots \mathcal{L}_{r}^{u_{r}}
$$

is positive.

\section{Applications to dual varieties of homogeneous spaces}

Let $G$ be a reductive group and let $P$ be a parabolic subgroup. Let $\Phi$ denote the root system of $G$, let $\Delta$ be a basis of $\Phi$ and let $\Delta^{\prime}$ be the subset of $\Delta$ corresponding to $P$. Let $\omega_{1}, \ldots, \omega_{s}$ be the set of fundamental weights corresponding to the roots from $\Delta^{\prime}$. For any weight $\lambda$ we will denote by $\mathcal{L}(\lambda)$ the corresponding line bundle on $G / P$. It is well known that the bundles $\mathcal{L}\left(\omega_{1}\right), \ldots, \mathcal{L}\left(\omega_{s}\right)$ generate the Picard group of $G / P$. Moreover, very ample line bundles on $G / P$ are the bundles $\mathcal{L}(\lambda)$ where $\lambda=\sum_{i=1}^{s} m_{i} \omega_{i}$, where $m_{i}>0$ for $i=1, \ldots, s$. We denote by $\rho_{P}$ the weight $\sum_{i=1}^{s} \omega_{i}$. Let $\lambda=\sum_{i=1}^{s} m_{i} \omega_{i}$ be a very ample line bundle on $G / P$. Let $X(\lambda)$ denote the variety $G / P$ embedded in the projective space by the embedding corresponding to $\lambda$. We are interested in the degree of the variety $X(\lambda)^{\vee}$. Let us denote this degree by $d(\lambda)^{\vee}$.

Finally we recall Kleiman's formula $([\mathrm{Kl}](\mathrm{IV}, 64)$ or $[\mathrm{K}]$, section 5$)$. It says that if $X$ is a smooth projective variety of dimension $l$ in $P^{n}$ and if $\mathcal{L}=\left.O_{P^{n}}(1)\right|_{X}$, then

$$
d\left(X^{\vee}\right)=\sum_{i=0}^{l}(i+1) \int_{X} c_{l-i}\left(\Omega_{X}^{1}\right) \mathcal{L}^{i} .
$$

In our situation it follows that $d(\lambda)^{\vee}$ is a polynomial in $m_{1}, \ldots, m_{s}$.

Let us write $\lambda=\lambda^{\prime}+\rho_{P}$. Let us denote $\lambda^{\prime}=\sum_{i=1}^{s} m_{i}^{\prime} \omega_{i}$, where $m_{i}^{\prime}=m_{i}-1$ for each $i$ and therefore $m_{i}^{\prime} \geq 0$ for each $i$. We can certainly find a polynomial $Q\left(m_{1}^{\prime}, \ldots, m_{s}^{\prime}\right)$ such that $d(\lambda)^{\vee}=Q\left(m_{1}^{\prime}, \ldots, m_{s}^{\prime}\right)$. Our main application is

Theorem 3. The polynomial $Q\left(m_{1}^{\prime}, \ldots, m_{s}^{\prime}\right)$ has nonnegative coefficients.

Proof. We write $d(\lambda)^{\vee}=P\left(m_{1}, \ldots, m_{s}\right)$ where $P\left(m_{1}, \ldots, m_{s}\right)$ is a polynomial in $m_{1}, \ldots, m_{s}$. Then

$$
Q\left(m_{1}^{\prime}, \ldots, m_{s}^{\prime}\right)=P\left(m_{1}^{\prime}+1, \ldots, m_{s}^{\prime}+1\right) .
$$


We apply Proposition 1 to $\mathcal{L}=\rho_{P}, \mathcal{L}_{i}=\omega_{i}$. It is well known that the assumptions of Proposition 1 are satisfied for these bundles. Now the Taylor formula for the values of $Q$ and Proposition 1 imply Theorem 3.

Examples. (1) Let $G=S L(n)$ and let $P$ be the stabilizer of the line in the $n$ dimensional space. Then $G / P=P^{n-1}$ and $\lambda=m \omega_{1}$. The degree of $X(\lambda)^{\vee}$ is equal to the degree of the discriminant of a homogeneous polynomial of degree $m$ in $n$ variables. Thus $d(m)^{\vee}=n(m-1)^{n-1}$. Substituting $m^{\prime}=m-1$ we see that $d\left(m^{\prime}\right)^{\vee}=n\left(m^{\prime}\right)^{n-1}$, so it is a polynomial with nonnegative coefficients.

(2) Let $G=S L(3)$ and let $P=B$ be the Borel subgroup. The weight $\lambda$ can be written as $\lambda=m_{1} \omega_{1}+m_{2} \omega_{2}$. It is easy to see using Kleiman's formula that

$$
d(\lambda)^{\vee}=12\left(m_{1}^{2} m_{2}+m_{1} m_{2}^{2}\right)-6\left(m_{1}^{2}+4 m_{1} m_{2}+m_{2}^{2}\right)+12\left(m_{1}+m_{2}\right)-6 .
$$

Substituting $m_{1}^{\prime}=m_{1}-1$ and $m_{2}^{\prime}=m_{2}-1$ we see that

$$
\begin{aligned}
d(\lambda)^{\vee}= & 12\left(\left(m_{1}^{\prime}\right)^{2} m_{2}^{\prime}+m_{1}^{\prime}\left(m_{2}^{\prime}\right)^{2}\right)+6\left(\left(m_{1}^{\prime}\right)^{2}+4 m_{1}^{\prime} m_{2}^{\prime}+\left(m_{2}^{\prime}\right)^{2}\right) \\
& +12\left(m_{1}^{\prime}+m_{2}^{\prime}\right)+6 .
\end{aligned}
$$

We will denote the polynomial $Q$ constructed above by $Q_{G, P}$. It is an interesting (but probably very difficult) problem to find the combinatorial description of the coefficients of polynomials $Q_{G, P}$.

We finish this section with two quick applications of Theorem 3.

Corollary 4. Let us assume that $\lambda$ satisfies the condition $m_{i} \geq 2$ for $i=1, \ldots, s$. Then $d(\lambda)^{\vee}>0$, i.e., the variety $X(\lambda)^{\vee}$ is a hypersurface.

Proof. Our assumption means that $m_{i}^{\prime}>0$ for each $i$. This means that the value of the highest term of $Q$ evaluated at $\lambda$ is positive. This, together with Theorem 3 , proves the corollary.

Corollary 4 was proven in the paper of Knop and Mentzel [K-M] by a different method.

Corollary 5. Let $G$ be a simple group. Then for any positive number $N$ there are only finitely many dominant integral weights $\lambda$ such that $d(\lambda)^{\vee} \leq N$.

Proof. It is enough to prove this statement for the weights $\lambda$ corresponding to the embeddings of the fixed $G / P$. If $X(\rho)^{\vee}$ is a hypersurface, then by [K], Proposition $3.5, \phi$ is a birational isomorphism. Thus for each monomial $\mathcal{L}\left(\omega_{1}\right)^{u_{1}} \cdots \mathcal{L}\left(\omega_{r}\right)^{u_{r}}$ we can choose the smooth representative $Y$ of this class, so $\operatorname{dim} \phi_{*}\left(P_{Y}\right)=\operatorname{dim} P_{Y}$. By Corollary 2 (applied to $\mathcal{L}=\rho_{P}, \mathcal{L}_{i}=\mathcal{L}\left(\omega_{i}\right)$ ) we see that each monomial in $Q\left(m_{1}^{\prime}, \ldots, m_{s}^{\prime}\right)$ occurs with a positive coefficient. The result follows immediately. Now let us assume that $\operatorname{codim} X\left(\rho_{P}\right)^{\vee}>1$. Then by the classification of KnopMentzel $([\mathrm{K}-\mathrm{M}])$ we see that $P$ is a maximal parabolic subgroup, so $s=1$. Then $Q\left(m_{1}^{\prime}\right)$ is a polynomial of positive degree, and the result follows.

\section{The degree of the dual variety to $G / B$ and permanents}

In this section we investigate the constant term of the polynomial $Q_{G, P}$ in the case when $P=B$ is a Borel subgroup of $G$. By definition this term is the degree $d(\rho)^{\vee}$ of the variety $X(\rho)^{\vee}$. By Kleiman's formula this degree equals

$$
d(\rho)^{\vee}=\sum_{s}(N-s+1) \int_{G / B} c_{s}(G / B) \rho^{N-s} .
$$


Therefore we want to compute the terms $\int_{G / B} c_{s}(G / B) \rho^{N-s}$. These terms have a nice description in terms of permanents of certain matrices associated to root systems.

Before we start our calculations we change our setup slightly.

Since $G / B$ has a cellular decomposition into locally closed cells (Schubert cells), the Chow ring $A^{\bullet}(G / B)$ is canonically isomorphic to the cohomology ring.

We can therefore make our computation in the cohomology ring. We can also assume we work with cohomology with rational coefficients, since we are going to compute integrals of forms (or make computations of degree).

Now let $G$ be a topological group; denote by $B G$ its classifying space, and by $E \rightarrow B G$ the classifying fibration. By definition of equivariant cohomology we have

$$
H_{G}^{*}(X, Q)=H^{*}\left(E \times_{G} X, Q\right) .
$$

Now let us take $G$ to be a semisimple simply connected group, $T$ a maximal torus, $B \supset T$ a Borel subgroup and $X=G / B$. We also denote by $K$ a maximal compact subgroup of $G$ such that $T_{K}=T \cap K$ is a maximal torus in $K$ so that $G / B=K / T_{K}$. Then $E \times_{G} X \equiv E \times_{K} X$ (三 means homotopy equivalent) since $G / K$ is contractible. But $E \times_{K} X=E / T_{K}=B T_{K}$.

Choosing a basis of the character ring of $T_{K}$, we get an isomorphism of this group with $\left(S^{1}\right)^{r}$, where $r=\operatorname{dim} T=\operatorname{rank} G$. Now $B S^{1} \equiv P^{\infty}$, so $H^{*}\left(P^{\infty}, Q\right)=Q[x]$, where $x$ is the hyperplane class.

It follows immediately that

$$
H_{G}^{*}(X, Q)=H^{*}\left(B T_{K}, Q\right)=S^{*}\left(X(T) \otimes_{Z} Q\right),
$$

since $B\left(S^{1}\right)^{r}=\left(B S^{1}\right)^{r}$.

Now it is well known that $H^{*}(B G, Q)=H^{*}(B K, Q)-H^{*}\left(B T_{K} Q\right)^{W}, W$ being the Weyl group (compare [B]).

Now consider the spectral sequence of the fibration $B T_{K} \rightarrow B K$ with fiber $X$. All spaces involved (total, base, fiber) have only cohomology in even degree, so the spectral sequence collapses at the $E_{2}$ term. We deduce that

$$
H^{*}(X, Q)=H^{*}\left(B T_{K}, Q\right) / I
$$

where $I$ is the ideal generated by the elements of positive degree in $H^{*}(B K, Q)$, i.e., by the positive degree $W$-invariants in $S^{*}\left(X(T) \otimes_{Z} Q\right)$, as desired.

The lattice $X(T)$ can be identified with the weight lattice $\Lambda$. Denoting $\Lambda_{Q}=$ $\Lambda \otimes Q$ we can rewrite (13) and (14) as

$$
\begin{gathered}
H_{G}^{*}(X, Q)=\operatorname{Sym}\left(\Lambda_{Q}\right), \\
H^{*}(G / B, Q)=H_{G}^{*}(G / B, Q) / I,
\end{gathered}
$$

where $I$ is the ideal generated by $W$-invariant elements of positive degree.

Let $\lambda \in \Lambda$. Then we denote the equivariant Chern class of the line bundle $\mathcal{L}(\lambda)$ which admits a canonical $G$-linearisation by $\lambda$.

For any coroot $\alpha^{\vee} \in \Lambda_{Q}^{*}$ we define the operator $D_{\alpha} \vee$ on $H_{G}^{*}(G / B, Q)$ by

$$
D_{\alpha^{\vee}}(\lambda)=\left\langle\alpha^{\vee}, \lambda\right\rangle \quad \text { for all } \lambda \in \Lambda_{Q} \text {. }
$$

We consider now the differential operator on $H_{G}^{*}(G / B, Q)$ :

$$
D=\frac{\prod_{\alpha>0} D_{\alpha \vee}}{\prod_{\alpha>0}\left(\alpha^{\vee}, \rho\right)}
$$


Let $N$ be the number of positive roots in $\Phi$. Then the operator $D$ decreases the degree by $N$, so in particular we get a linear form

$$
D: H_{G}^{N}(G / B, Q) \rightarrow H_{G}^{0}(G / B, Q)=Q .
$$

Proposition 6. Let $x \in H^{N}(G / B, Q)$. Then

$$
\int_{G / B} x=D \tilde{x}
$$

where $\tilde{x} \in H_{G}^{N}(G / B, Q)$ is a lifting of $x$.

Proof. Every simple reflection $s_{i} \in W$ permutes the positive coroots other than $\alpha_{i}^{\vee}$ and $s_{i} \alpha_{i}^{\vee}=-\alpha_{i}^{\vee}$. Therefore we deduce that if $y \in H_{G}^{N}(G / B, Q)$, then $D(w y)=$ $(-1)^{l(w)} D(y)$. In particular $D$ kills every irreducible component of $H_{G}^{N}(G / B, Q)$ not isomorphic to the sign representation. Since the kernel $K$ of the map

$$
H_{G}^{N}(G / B, Q) \rightarrow H_{G}^{0}(G / B, Q)
$$

is $W$-stable and contains no irreducible representation isomorphic to the sign representation, we have

$$
\left.D\right|_{K}=0 .
$$

This means that $D \tilde{x}$ does not depend on the choice of $\tilde{x}$ and that

$$
D \tilde{x}=a \int_{G / B} x .
$$

To finish the proof it is enough to show that $a=1$. For this we consider the bundle $\mathcal{L}(\rho)$. This is a very ample bundle on $G / B$, and by the Weyl dimension formula we have

$$
h^{0}\left(G / B, \mathcal{L}(\rho)^{\otimes n}\right)=(n+1)^{N} .
$$

It follows that (again denoting by $\rho$ the Chern class $c_{1}(\mathcal{L}(\rho))$ )

$$
\int_{G / B} \rho^{N}=N !
$$

On the other hand, calculating directly from the definition we see that

$$
D \rho^{N}=N !
$$

so $a=1$ and the proposition is proved.

We now want to compute

$$
\int_{G / B} c_{s}(G / B) \rho^{N-s}
$$

Let us recall that

$$
c_{s}(G / B)=\sum_{\Gamma \subset \Phi^{+}, \operatorname{card}} \prod_{\Gamma=s} \alpha .
$$

By Proposition 6 we need to compute

$$
D\left(\sum_{\Gamma \subset \Phi^{+}, \operatorname{card} \Gamma=s}\left(\prod_{\alpha \in \Gamma} \alpha\right) \rho^{N-s}\right)=(N-s) ! \sum_{\Gamma \subset \Phi^{+}, \Delta \subset \Phi^{+\vee}} \prod_{\alpha \in \Gamma, \beta^{\vee} \in \Delta} \frac{\left(\beta^{\vee}, \alpha\right)}{\left(\beta^{\vee}, \rho\right)} .
$$


We can summarize this result in the following way. Fix an order $\alpha_{1}, \ldots, \alpha_{N}$ on $\Phi^{+}$and $\beta_{1}^{\vee}, \ldots, \beta_{N}^{\vee}$ on $\Phi^{\vee+}$. Let us define the $N \times N$ matrix

$$
B=\left(\frac{\left(\beta_{i}^{\vee}, \alpha_{j}\right)}{\left(\beta_{i}^{\vee}, \rho\right)}\right)_{1 \leq i, j \leq N} .
$$

Then we have

\section{Proposition 7.}

$$
\int_{G / B} c_{s}(G / B) \rho^{N-s}=(N-s) ! P_{s}(B)
$$

where $P_{s}(B)$ is the sum of all the permanents of $s \times s$ submatrices of the matrix $B$.

\section{ACKNOWLEDGMENT}

This work was done during the second author's stay at the University Roma II and at Scuola Normale Superiore di Pisa in the fall of 1992. He would like to thank the C.N.R.S. and Scuola Normale for support during that period.

\section{REFERENCES}

[B] A. Borel, Cohomologie des espaces fibrés principaux, Ann. Math. 57 (1953), 115-207. MR 14:490e

[G-K-Z] I. M. Gelfand, M. M. Kapranov, A. V. Zelevinski, A-discriminants and the Cayley-Koszul complexes, Dokl. Akad. Nauk SSSR 307 (1989), 1307-1311. (Russian) MR 90k:14054

[Ha] R. Hartshorne, Algebraic Geometry, Graduate Texts in Mathematics, No. 52, SpringerVerlag, New York-Heidelberg-Berlin, 1977. MR 57:3116

[K] N. Katz, Pinceaux de Lefschetz; Theoreme d'existence, in: SGA 7, Lecture Notes in Math., vol. 340, pp. 212-253.

[Kl] S. Kleiman, Enumerative theory of singularities, Real and Complex Singularities (Proc. Ninth Nordic Summer School/NAVF Sympos. Math., Oslo, 1976), pp. 297-396. MR 58:27960

[K-M] F. Knop, G. Mentzel, Duale Varietäten von Fahnenvarietäten, Comm. Math. Helv. 62 (1987), 38-61. MR 89a:14051

[W] J. Weyman, Calculating discriminants by higher direct images, Trans. Amer. Math. Soc. 343 (1994), 367-389. MR 94g:14026

Department of Mathematics, Scuola Normale Superiore, Pisa, Italy

E-mail address: deconcin@ux1sns.sns.it

Department of Mathematics, Northeastern University, Boston, Massachusetts 02115

E-mail address: weyman@neu.edu 